\title{
Sand - rubber mixtures submitted to isotropic loading: a minimal model
}

\author{
Auriane PLATZER ${ }^{1,2, \star}$, Salman ROUHANIFAR ${ }^{3, \star \star}$, Patrick RICHARD ${ }^{1, \star \star \star}$, Bogdan CAZACLIU ${ }^{1, \star \star \star \star}$, and Erdin \\ IBRAIM ${ }^{3, \dagger}$ \\ ${ }^{1}$ LUNAM Université, IFSTTAR, MAST, GPEM, F-44340 Bouguenais, France \\ ${ }^{2}$ GeM (Institut de recherche en génie civil et mécanique), CNRS UMR 6183, École Centrale de Nantes, F- 44321 Nantes, France \\ ${ }^{3}$ Department of Civil Engineering, University of Bristol. Bristol, BS8 1TR, UK
}

\begin{abstract}
The volume of scrap tyres, an undesired urban waste, is increasing rapidly in every country. Mixing sand and rubber particles as a lightweight backfill is one of the possible alternatives to avoid stockpiling them in the environment. This paper presents a minimal model aiming to capture the evolution of the void ratio of sand-rubber mixtures undergoing an isotropic compression loading. It is based on the idea that, submitted to a pressure, the rubber chips deform and partially fill the porous space of the system, leading to a decrease of the void ratio with increasing pressure. Our simple approach is capable of reproducing experimental data for two types of sand (a rounded one and a sub-angular one) and up to mixtures composed of $50 \%$ of rubber.
\end{abstract}

\section{Introduction}

The number of scrap tyres is increasing rapidly in both developed and developing countries. Consequently, the accumulation of used tyres is becoming a real societal problem. In this context, the possibility of recycling and mixing rubber chips derivatives with granular soil particles can be a solution for some geotechnical applications like backfilling for retaining structures, slope and highway embankment stabilization, road constructions, soil erosion prevention and seismic isolation of foundations [1]. In addition to their remarkable mechanical properties [2], such soilrubber chip composite mixtures have interesting acoustical and drainage properties too [3].

While their potential range of applications is wide, a full understanding of their behaviour, including internal interaction mechanisms resulting from the combination of two particular materials, one soft, tyre chip rubber, and one rigid, granular soil, deserves further studies despite recent interesting works [5-7]. Here, we present a theoretical model aiming to capture the evolution of the void ratio of sand-rubber mixtures of various volume ratios of rubber undergoing an isotropic compression loading. For the sake of simplicity, we focus on mixtures composed of rubber and sand particles of similar particle size distributions. Our model is based on the idea that, submitted to a pressure, the rubber chips deform and partially fill the porous space of the system leading to a void ratio which decreases with increasing pressure. Assuming the sand particles as infinitely rigid and the rubber particles as incompressible,

\footnotetext{
^e-mail: auriane.platzer@eleves.ec-nantes.fr

$\star \star$ e-mail: salman.rouhanifar@bristol.ac.uk

$\star \star \star$ e-mail: patrick.richard@ifsttar.fr

$\star \star \star \star$ e-mail: bogdan.cazacliu@ifsttar.fr

†e-mail: erdin.ibraim@bristol.ac.uk
}

our approach -similar to mean-field approaches- leads to an expression of the void ratio of the mixture as a function of: (i) the fraction of rubber that deforms and fills the porous space, (ii) the void ratio for the soil without rubber chips and (iii) the volume ratio of rubber. Assuming (i) varies exponentially with the applied pressure, we compare the predictions of our model with experimental test results and discuss its limits. Despite its simplicity, our approach successfully reproduces experimental data for two types of sand (a rounded one and a sub-angular one) and up to mixtures composed of $50 \%$ of rubber.

\section{Model}

To derive our model we consider granular packings made of sand grains and rubber chips. For the sake of simplicity, we focus here on packings for which all the particles have the same size distribution independently of their type (sand or rubber). We also consider an isotropic compression of the materials and the evolution with the pressure of the void ratio i.e. the ratio between volume of the porous space and volume of the solids. Intuitively, a simple linear combination of the respective void ratios according to the proportion of rubber is not relevant. Indeed, such an approach assumes the absence of correlation between the two phases. This is clearly not the case here, due to the potential important deformation of the rubber particles which affects the structure of the whole system. The volume ratio of rubber, $x_{R}$ is defined as the ratio $V_{R} /\left(V_{R}+V_{S}\right)$ where $V_{R}$ and $V_{S}$ are respectively the volume of rubber and that of sand. Our model is based on the following assumptions:

1. sand grains are infinitely rigid,

2. rubber chips are incompressible, 


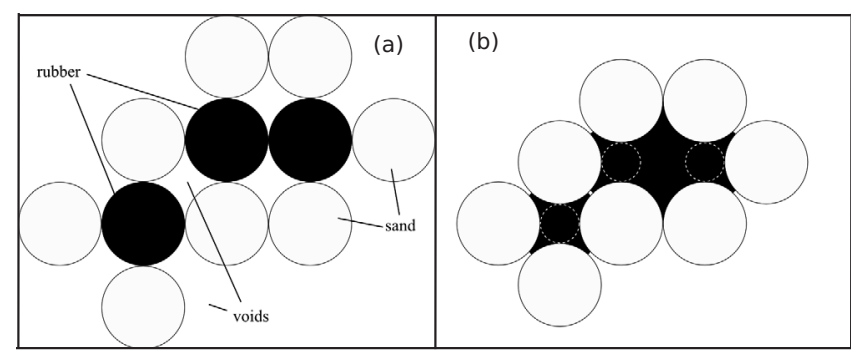

Figure 1. Sketch explaining our fourth assumption (see text). We assume that, due to the deformation of rubber particles, the porous space of an initially undeformed packing (a) is partially filled by the rubber (b).

3. the repartition of rubber chips is spatially homogenous,

4. we neglect particle rearrangements, i.e. the particles' neighborhood do not change. We just consider that the rubber chips undergo deformation due to pressure and fill the porous space (see figure 1). Such an assumption is fully consistent with the work of Feng and Sutter [4], in which they treat the volume of rubber chips as voids to estimate the maximum shear modulus.

5. Finally, we restrict ourselves to pressures important enough $\left(p>p^{*}=100 \mathrm{kPa}\right)$ to probe the properties of the granular material, not those of the grain surface. To estimate this threshold, we considered two $D$-diameter spherical sand grains interacting through the Hertz law. The macroscopic pressure necessary to achieve a deformation of the order of magnitude of the surface's asperities $(\delta \approx 100 \mathrm{~nm})$ is $p^{*}=\kappa \delta^{3 / 2} / D^{3 / 2}$, where $\kappa$ is a constant depending on the Young modulus $E$ and on the Poisson ratio $v$ through $\kappa=4 E /\left[3 \pi\left(1-v^{2}\right)\right]$. Using $E=90 \mathrm{GPa}$, $v=0.4$ leads to $p^{*} \approx 100 \mathrm{kPa}$ for $D$ of a few tenths of millimeter.

Note that, due to the two first assumptions, the volume ratio of rubber, $x_{R}$, is constant whatever the loading conditions. Let us now define a volumetric function $f$ which relates the total volume of rubber that fills the porous space to the total volume of rubber. This fraction, called the deformed fraction of rubber, depends on $x_{R}$ and on the pressure $p$. In the following we will derive an expression of $f$ relating this quantity to $x_{R}$ and $p$. For a given $x_{R}$, the evolution of deformed fraction of rubber, $f$, with the pressure is intuitive : at the early stages of the compression process the pores are large and easy to fill. Consequently, $f$ increases with the pressure. As the pores get filled, it becomes more and more difficult to fill the remaining pores and $f$ reaches a constant value. The simplest way to model such an increase towards a saturation value is to assume that $f$ obeys the following first order differential equation

$$
\left.p_{0}\left(x_{R}\right) \frac{\partial f}{\partial p}\right|_{x_{R}}+f\left(x_{R}, p\right)=F\left(x_{R}\right)
$$

where $p_{0}\left(x_{R}\right)$ is a characteristic pressure and $F\left(x_{R}\right)$ the maximum deformed fraction of rubber for an infinite pressure, i.e $f\left(x_{R}, p \rightarrow \infty\right)$. Such kind of equation are classically used to model systems which involve a driving force and viscous-like force. For example the fall of an object in a viscous fluid (the system is driven by gravity and slowed down by viscosity) or the loading of a capacitor (the system is driven by an electromotive force and slowed down by the Ohm law). Here, the system is driven by the compression and slowed down by the ability of the porous space to resist to the rubber filling (a permeabilitylike quantity). The solution of the preceding equation is

$$
f\left(x_{R}, p\right)=f^{*}+\left(F\left(x_{R}\right)-f^{*}\right)\left[1-\exp \left(-\frac{p-p^{*}}{p_{0}\left(x_{R}\right)}\right)\right],
$$

where $f^{*}=f\left(x_{R}, p=p^{*}\right)$. Since we restrict ourselves to pressures greater than $p^{*}$, it is more natural to use $\tilde{f}=$ $f-f^{*}$ and $\tilde{p}=p-p^{*}$ instead of respectively $f$ and $p$. Then:

$$
\tilde{f}\left(x_{R}, \tilde{p}\right)=\left(F\left(x_{R}\right)-f^{*}\right)\left[1-\exp \left(-\frac{\tilde{p}}{p_{0}\left(x_{R}\right)}\right)\right] .
$$

The parameters $p_{0}\left(x_{R}\right)$ and $F\left(x_{R}\right)$ are unknown functions of $x_{R}$ that can be determined from experiments. Indeed, $\partial \tilde{f} / \partial \tilde{p}$ is an affine function of the pressure $\tilde{p}$ whose intercept and slope are respectively given by $\ln \left[\left(F\left(x_{R}\right)-f^{*}\right) / p_{0}\left(x_{R}\right)\right]$ and $-1 / p_{0}$. Although it is probably possible to measure $f\left(x_{R}, p\right)$ using X-ray tomography at several stages of the compression process such an experimental campaign would be long and fastidious. Yet, it is possible to test our model by studying the evolution of the void ratio with pressure. As mentioned above, the void ratio is classically defined as $e=V_{V} / V_{\text {sol }}$, where $V_{V}$ and $V_{\text {sol }}$ are respectively the volume of the porous space and the volume of solids (i.e. the volume of the rubber chips and of the sand grains). Contrary to the former volume, the latter depends neither on the volume ratio of rubber, nor on the pressure. The volume of the porous space decreases with increasing pressure due to the gradual filling of the pores by the deformed rubber following the equation

$$
V_{V}\left(x_{R}, p\right)=V_{V}\left(x_{R}=0, p\right)-f\left(x_{R}, p\right) x_{R} V_{s o l} .
$$

Equivalently, the void ratio is given by

$$
e\left(x_{R}, p\right)=e\left(x_{R}=0, p\right)-f\left(x_{R}, p\right) x_{R} .
$$

Therefore, our model predicts that the void ratio decreases with the pressure according to the following equation:

$$
\begin{aligned}
e\left(x_{R}, p\right)= & e\left(x_{R}=0, p\right)-x_{R} f^{*} \\
& -x_{R}\left(F\left(x_{R}\right)-f^{*}\right)\left[1-\exp \left(-\frac{p-p^{*}}{p_{0}\left(x_{R}\right)}\right)\right] .
\end{aligned}
$$

This prediction requires the knowledge of (i) the evolution of the void ratio versus the pressure for a $100 \%$ sand sample (ii) the characteristic pressure, $p_{0}$, and (iii) the maximal deformed fraction of rubber, $F$. Since these three prerequisites depend on the type of sand used, it is necessary to test our model using at least two types of sand. 


\section{Comparison with experiments}

Two silica based uniform sand materials, Leighton Buzzard fraction A characterised as a coarse material (mean grain diameter, $D_{50}=1.6 \mathrm{~mm}$ ) and Hostun RF characterised as fine material $\left(D_{50}=0.38 \mathrm{~mm}\right)$, have been chosen for this study. The former sand is natural, the latter is industrially produced by crushing. The rubber particles are obtained from the shredding of used lorry tires and consist of polymer, acetone, carbon black, ash and Sulphur. Their Young modulus is approximately 3.5MPa. Following a tedious process that involved washing, drying, sorting and sieving, equivalent rubber granular materials have been engineered to match the particle size distributions of the two sands. The fabrication process of all samples of cylindrical shape (70 $\mathrm{mm}$ diameter and $70 \mathrm{~mm}$ height) is done using the moist tamping. The method implies the succession of three stages: mixing, deposition and compaction. Sand and rubber particles are mixed by adding $10 \%$ water content and the mixture is placed into the triaxial cylindrical mould in three successive layers. Each layer is compacted up to a pre-definite height using a circular tamper with a diameter being half of the sample diameter. While this technique appears to be more effective in discouraging segregation of the composite constituents, providing good control of sample density and homogeneous distribution of rubber, it also produces a soil-rubber fabric which may correspond to that obtained in rolledcompacted construction fills. Once the sample is fabricated, a top cap together with a lateral latex membrane seal the sample and a small vacuum of $20 \mathrm{kPa}$ is applied. This vacuum is necessary for the protection of sample's fabric and stability during removal of the cylindrical mould. At this stage, the mixtures have initial void ratios which are reported in table 1 for various volume ratios of rubber $x_{R}=0.1,0.2,0.3,0.5$ and 1 . The triaxial cell con-

Table 1. Initial void ratios of the samples

\begin{tabular}{cc|cc}
\hline \multicolumn{2}{c|}{$\begin{array}{c}\text { Leighton Buzzard } \\
\text { Sand-Rubber Mixtures }\end{array}$} & \multicolumn{2}{c}{$\begin{array}{c}\text { Hostun } \\
\text { Sand-Rubber Mixtures }\end{array}$} \\
\hline$x_{R}$ & void ratio & $x_{R}$ & void ratio \\
\hline 0. & 0.630 & 0. & 0.746 \\
0.05 & 0.627 & 0.05 & 0.741 \\
0.1 & 0.625 & 0.1 & 0.739 \\
0.2 & 0.620 & 0.2 & 0.736 \\
0.3 & 0.618 & 0.3 & 0.736 \\
0.5 & 0.604 & 0.5 & 0.719 \\
1. & 0.602 & 1. & 0.707
\end{tabular}

taining the cylindrical sample is then filled with water and pressurized to a pressure of $30 \mathrm{kPa}$ while the vacuum is released. All samples are saturated by applying $\mathrm{CO}_{2}$ for around 45 minutes followed by flushing de-aired water to obtain Skempton coefficient $(B)$ higher than 95\%. A backpressure of $100 \mathrm{kPa}$ is normally used. Both the back pressure and cell (total pressure) pressure are measured by pressure transducers. The samples are then loaded isotropically up to the maximum pressure of $550 \mathrm{kPa}$ at which point, an unloading stage is applied. During the isotropic
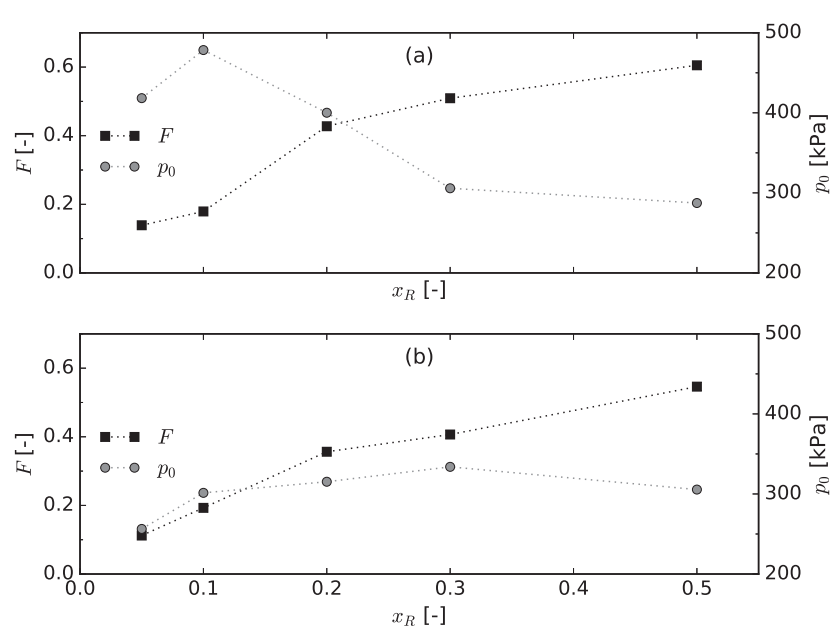

Figure 2. The parameters $F$ and $p_{0}$ ((a): Hotsun sand (b) : Leighton Buzzard sand) are obtained by fitting experimental data

compression, the sample volumetric changes are measured by a volume change measurement device accounting for the amount of water that is expelled in the process.

We have reported in figure 2 the values of the parameters $p_{0}$ and $F$ versus the volume ratio of rubber for mixture made of rubber and Hotsun sand (a) and of rubber and Leighton Buzzard sand (b). Both quantities are obtained by fitting $\ln \partial \tilde{f} / \partial \tilde{p}$ by an affine function (see section 2 ). The characteristic pressure $p_{0}$ depends on the type of sand. Its evolution with $x_{R}$ is not clear since it is not monotonic. On the contrary, $F$ (the maximum fraction of deformed rubber) increases with $x_{R}$ but seems to reach a plateau.

To compare directly the experimental results with the prediction of our model we report in figure 3 (for rubberHotsun sand mixtures) and in figure 4 (for rubber-Leighton Buzzard mixtures) the experimental void ratios versus the applied pressure as well as $\tilde{e}$, the void ratios determined by our theoretical predictions i.e.

$$
\tilde{e}\left(x_{R}, \tilde{p}\right)=e\left(x_{R}=0, \tilde{p}\right)-f\left(x_{R}, \tilde{p}\right) x_{R} .
$$

A good agreement between our minimal model and experimental data is found for the two types of sand. This demonstrates that the main idea of our model i.e. the pores are partially filled by the deformed fraction of the rubber particles is reasonable, while neglecting the particle rearrangement effect. However, a full validation of our model is still necessary. In particular it would be interesting to calibrate $p_{0}$ and $F$ using other loading conditions.

\section{Discussion}

As mentioned above, our model is based on the idea that due to compression the rubber particles deform and partially fill the porous space of the system. Although the comparison made with experimental results seems to validate this idea, the study of the deformation of the rubber particles during the compression process (e.g. by Xray tomography or by numerical simulation coupling Discrete Element Method to model sand and finite element 


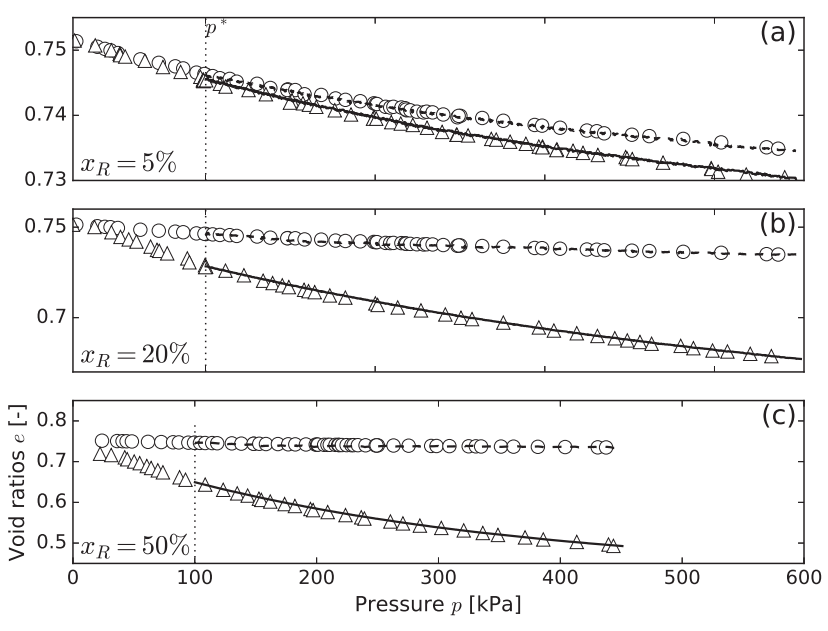

Figure 3. Comparison between experimental void ratios and those obtained by our model for several volume ratios of rubber and the Hotsun sand. Triangles (resp. circles) correspond to the sample with rubber $\left(e\left(x_{R}, p\right)\right)$ (resp. without rubber $e\left(x_{R}=0, p\right)$ and the solid line (resp. dotted line) to the prediction of our model $\tilde{e}\left(x_{R}, p\right)\left(\operatorname{resp} . \tilde{e}\left(x_{R}=0, p\right)\right)$.
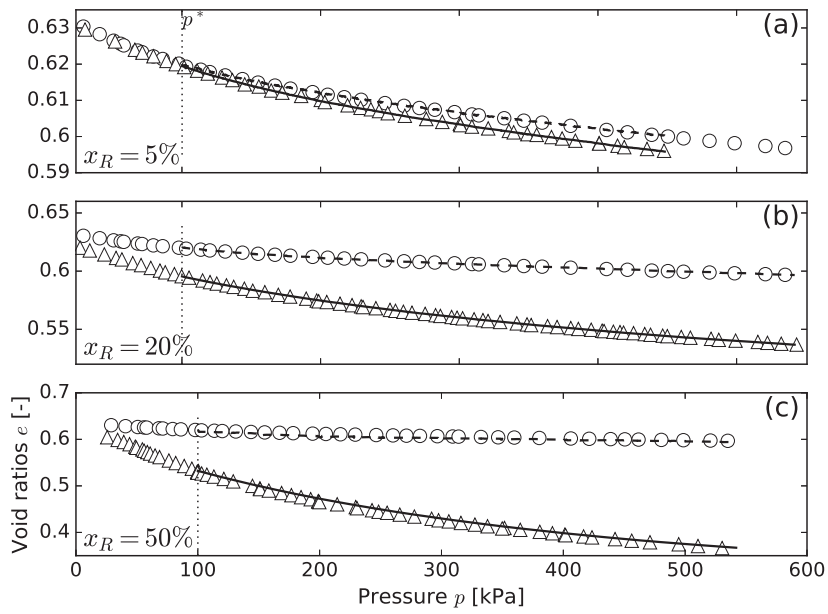

Figure 4. Comparison between experimental void ratios and those obtained by our model for several volume ratios of rubber and the Leighton Buzzard sand. Triangles (resp. circles) correspond to the sample with rubber $\left(e\left(x_{R}, p\right)\right)$ (resp. without rubber $e\left(x_{R}=0, p\right)$ and the solid line (resp. dotted line) to the prediction of our model $\tilde{e}\left(x_{R}, p\right)$ (resp. $\left.\tilde{e}\left(x_{R}=0, p\right)\right)$.

modelling of rubber) is necessary to fully validate our approach. In the absence of such a study we can discuss the assumptions we used and their validity. Among them, some are fully justified. For example the assumption that sand is infinitely rigid is justified by the value of the Young modulus of the sand which is three order of magnitude greater than that of rubber. Similarly, assuming the incompressibility of rubber is justified by its Poisson coefficient whose value is close to 0.5 . Other assumptions probably deserve deeper justifications. Firstly, the range of pressure studied. We indeed assume that our model is only valid for pressures greater than a threshold $p^{*}$ below which the deformation is too weak to neglect the importance of the surface properties of the grains. Relaxing this assumption would require to model the surface properties of both the Hotsun sand and the Leighton Buzzard sand and include them in the model, which is out of the scope of this paper. Secondly, we have neglected particle rearrangements (each particle keeps its neighbourhood). Although reasonable in the case of packing submitted to compression this is probably no more the case when the packings are submitted to shear. Thirdly, we have assumed that the rubber grains are homogeneously distributed within the sample. In other words, every rubber grain has, statistically, the same neighbourhood. Clusters are allowed but their probability of existence have to be independent of their position.

Finally, it is worth mentioning three main perspectives of this work. First, what happens for volume ratios of rubber greater than 0.5? Is our model still valid? Second, how can we adapt our model for packings where sand and rubber particles have different size distributions? Third, as mentioned above, a direct measurement of the deformed fraction of rubber $f$ would be an ultimate test for our model.

\section{Conclusions}

We derived a model aiming to predict the behaviour of mixtures of sand grains and rubber chips undergoing isotropic compression. We restricted ourselves to mixtures for which the size distributions of the sand and rubber particles are similar. Our model assumes that the deformed fraction of rubber, which increases with the pressure, fills the porous space and that its evolution with the pressure obeys a first order equation whose parameters can be determined experimentally. The experimental results are captured in a satisfactory way. The next step of this study is to adapt our model to the cases where the sand particles and the rubber chips significantly differ in size.

\section{References}

[1] Lee, J. H.; Salgado, R.; Bernal, A. and Lovell, C. W., Journal of Geotechnical and Geoenvironmental Engineering, 125, 132-141 (1999)

[2] Youwai, S. and Bergado, D. T., Canadian Geotechnical Journal, 40, 254-264 (2003)

[3] Valdes, J. R. and Evans, T. M., Canadian Geotechnical Journal, 45, 588-595, (2008)

[4] Feng, Z. and Sutter, K., Geotechnical Testing Journal, 23, 338-344 (2000)

[5] Eidgahee, D. R. and Hosseininia, E. S., AIP Conference Proceedings, 1542, 269-272 (2013)

[6] Perez, J. L.; Kwok, C. and Senetakis, K., Computers and Geotechnics , 80, 199 - 214, (2016)

[7] Lee, C.; Shin, H. and Lee, J.-S., International Journal for Numerical and Analytical Methods in Geomechanics, 38, 1651-1663 (2014) 